\title{
Heritability Estimates for Antibody Response to Mycobacterium avium subspecies paratuberculosis in German Holstein Cattle
}

\author{
M. Hinger, H. Brandt, and G. Erhardt ${ }^{1}$ \\ Justus-Liebig University, Department of Animal Breeding and Genetics, Giessen, Germany
}

\begin{abstract}
The objective of this study was to estimate heritability of antibody response to Mycobacterium avium ssp. paratuberculosis (MAP) in 4,524 German Holstein cows by applying linear and threshold models. Data were collected within a paratuberculosis voluntary control program in Thuringia, Germany, in 2005. The MAPpositive prevalence of the 12 farms in the data set varied between 5 and $36.9 \%$. A nearly linear increase in prevalence was observed from 2- to 3-yr-old cows, whereas prevalence declined in cows older than $5 \mathrm{yr}$. This could be explained by greater culling rates associated with increasing age. Classification as MAP positive, questionable, and negative was available for all cows, and the optical density values of the Svanovir ELISA test existed for 2,084 of the animals originating from 6 farms. The heritability estimates of linear and threshold animal and sire models were compared. For the available data sets with an average of 8 progeny per sire, animal models were more robust and yielded more reliable results than did sire models. Heritability estimates from sire models led to overestimation of genetic variances because of a low number of progeny per sire and average relationship within sire progeny of greater than one-fourth, as expected between half-sibs. For all animal models, a heritability of about 0.1 was estimated for antibody response to MAP. Furthermore, it can be concluded that for the estimation of breeding values for antibody response to MAP optical density values of the ELISA test as a normally distributed trait (log-transformed) should be used rather than MAP status (positive or negative) as a binary trait because of the greater heritability and more robust parameter estimates when sire or animal models are used.
\end{abstract}

Key words: paratuberculosis, cattle, heritability estimation

Received January 16, 2008.

Accepted May 5, 2008.

${ }^{1}$ Corresponding author: Georg.Erhardt@agrar.uni-giessen.de

\section{INTRODUCTION}

Paratuberculosis (Johne's disease) in ruminants is caused by Mycobacterium avium ssp. paratuberculosis (MAP; Clarke, 1997). Infection with MAP often occurs in calves through ingestion of contaminated feces, and clinical disease generally does not become apparent until 3 to 5 yr post-exposure. Fetal infection is also possible and emerges mostly in dams with advanced clinical disease (Sweeney, 1996). Signs of clinical disease are intermittent diarrhea, weight loss, and eventually death. In addition, reduced performance in milk production, reproductive problems, and culling of infected animals also affect dairy and beef cattle industries all over the world. Ott et al. (1999) estimated losses for the US dairy industry of $\$ 200$ to 250 million annually caused by subclinical MAP infection, where the main factor was reduction in milk production followed by net cow replacement costs. Infection by MAP is not treatable and vaccination against MAP does not prevent infection (Chiodini et al., 1984).

The apparent herd prevalence of MAP infection varies between countries. In the United States, the USDA's National Animal Health Monitoring System (NAHMS, 1997) reported an apparent herd-level prevalence of $21.6 \%$ across all herd sizes. In contrast, Hacker et al. (2004) found $84.7 \%$ positive dairy herds in Mecklenburg-Westpommerania, Germany. In Denmark 8.8\% of dairy cows tested positive (Jakobsen et al., 2000), whereas Muskens et al. (2000) found 54.7\% of all tested Dutch dairy herds to have positive animals. For Austria, Gasteiner et al. (1999) reported 6.97\% positive cattle herds. In this study, the 4 oldest cattle in a herd were examined. Dreier et al. (2006) found that 19\% of dairy cattle were positive for antibodies against MAP, $10.1 \%$ had a questionable result, and $70.9 \%$ of all tested animals were antibody negative in their sample of 756 cattle from abattoirs all over Austria, each representing one herd. This is less than in other countries, but suggests that the apparent prevalence of MAP infection has increased in Austria compared with results published 7 yr earlier (Gasteiner et al., 1999). Unfortunately, both studies are not exactly comparable because of differing sampling procedures. 
Because Johne's disease is mainly influenced by environmental factors, the control of this disease has relied on reduction of transmission via hygiene programs. The success of such programs depends on individual farm management and is hard to standardize (Goodger et al., 1996; Muskens et al., 2003). Because of the lack of cost-effective, routinely applicable methods to detect infection in early stages, breeding programs seem to be an adequate method to increase herd health. Although numerous diagnostic tests for Johne's disease, including serum and milk ELISA and fecal bacterial culture, are available, low sensitivity and specificity limit the application of all these tests. The possibility of a sustainable decrease in disease prevalence through breeding for resistant animals depends on the heritability for resistance against MAP infection. Most traits concerning resistance toward infectious diseases are of low heritability (Heringstad et al., 2005; Snowder et al., 2005) and hard to influence by classical selection. On the other hand, marker-assisted selection allows progress in animal breeding through indirect selection methods (Dekkers and Hospital, 2002; Raadsma and Fullard, 2006).

A study by Gonda et al. (2007) showed a QTL for susceptibility to MAP infection on Bos taurus autosome 20 in US Holstein families. On the other hand, Hinger et al. (2007) could not detect a significant association between microsatellites located near or within candidate genes involved in immune response mechanisms and MAP infection status.

Koets et al. (2000) estimated heritabilities based on microbiological and histological postmortem examination of slaughtered cows. In nonvaccinated animals, heritability was $<0.01$, whereas the data set with known maternal phenotypes revealed heritability of 0.08 . In a Danish study, heritabilities of 0.09 with a sire model and 0.10 applying an animal model for ELISA test results of milk samples was estimated (Mortensen et al., 2004). Gonda et al. (2006) studied daughters of 12 sires tested for paratuberculosis infection with both ELISA and fecal culture. Considering data from both tests for trait definition resulted in a heritability estimate of 0.10 . The preceding studies used either data from a daughter design with only 12 highly selected sires involved (Gonda et al., 2006) or data from a vaccination trial (Koets et al., 2000). Only the study on Danish Holsteins (Mortensen et al., 2004) used a similar data structure with only linear models for ELISA test results.

Therefore, the aim of the present study was to estimate the genetic variation and heritability for paratuberculosis infection in German Holstein cows. Information about the MAP status of lactating cows from 2 different ELISA tests was used. Additionally, for all cows tested with the Svanovir ELISA test (Svanova Biotech AB, Uppsala, Sweden), the optical density (OD) values were available. The study was focused on the differences in heritability estimates between sire and animal models using MAP status on one hand and differences when using the MAP status as binary or normal distributed trait on the other hand.

\section{MATERIALS AND METHODS}

\section{Data Collection}

A total of 4,524 German Holstein cows originating from 12 farms routinely screened for MAP status within a voluntary control program in Thuringia (Germany) were available for the analyses as a random sample. The herd sizes varied from 139 to 655 cows tested.

The present study was based on test results from spring 2005. An antibody test on the serum samples was determined by commercially available ELISA tests (Svanovir ParaTB Ab ELISA Kit, Svanova or IDEXX HerdChek M. pt. Ab ELISA-Test Kit, IDEXX, Wörrstadt, Germany). In total, 2,878 of the animals were tested with the Svanovir assay, and 1,646 animals were tested with IDEXX test. Interpretation of sample results concerning the MAP status followed the manufacturer's instructions, respectively [IDEXX: S/P ratio = (OD of sample - OD of negative control)/(OD of positive control - OD of negative control), where $\geq 0.25=$ positive; $0.10-0.24=$ questionable; $\leq 0.09$ =negative; Svanovir: OD $>53 \%=$ positive; $52-32 \%=$ questionable; $<31 \%$ = negative .

The MAP status (negative, positive, or questionable) based on these results and full pedigree data were available for all cows tested (data set 1 ). In addition, the optical density values (ODV) of the Svanovir ELISA test were available for 2,084 of the cows originating from 6 farms (data set 2 ).

In Table 1, a summary of the 2 data sets is given, including number of sires, average number of daughters per sire, distribution of MAP status, and average optical density values. To obtain a normal distribution, the ODV were transformed as follows:

$$
\mathrm{ODV}_{\mathrm{T}}=\log _{\mathrm{e}}(\mathrm{ODV})
$$

\section{Statistical Analysis}

For MAP status and the $\mathrm{ODV}_{\mathrm{T}}$, a linear model $(\mathbf{L M})$ assuming normal distribution for the residual effect was implemented. The MAP status was additionally analyzed using a threshold model (TM) for binary traits. For this approach, 2 models were made, one where the variable "QPOS" included MAP-questionable animals as a positive outcome, and one where the 
Table 1. Description of the 2 data sets analyzed ${ }^{1}$

\begin{tabular}{|c|c|c|c|}
\hline Item & Data set 1 & & Data set 2 \\
\hline \multicolumn{4}{|l|}{ Cows, n } \\
\hline$\leq 2$ yr old & 1,053 & & 488 \\
\hline 3 yr old & 1,431 & & 827 \\
\hline 4 yr old & 919 & & 396 \\
\hline 5 yr old & 543 & & 181 \\
\hline 6 yr old & 293 & & 102 \\
\hline$>6$ yr old & 285 & & 90 \\
\hline Total & 4,524 & & 2,084 \\
\hline Farms, $\mathrm{n}$ & 12 & & 6 \\
\hline Sires, $\mathrm{n}$ & 564 & & 297 \\
\hline Daughters, ${ }^{2} \mathrm{n}$ (range) & $8.0(1$ to 161$)$ & & $7.0(1$ to 117$)$ \\
\hline MAP status, $\%$ & IDEXX or Svanovir & IDEXX & Svanovir \\
\hline Positive & 14.1 & 9.6 & 14.1 \\
\hline Questionable & 17.1 & 11.8 & 22.7 \\
\hline Negative & 68.8 & 78.6 & 63.2 \\
\hline
\end{tabular}

${ }^{1}$ Data set 1 = data with Mycobacterium avium ssp. paratuberculosis (MAP) status results from Svanovir (Svanovir ParaTB Ab ELISA Kit, Svanova Biotech AB, Uppsala, Sweden) and IDEXX (IDEXX HerdChek M. pt. Ab ELISA-Test Kit, IDEXX, Wörrstadt, Germany) test. Data set $2=$ data from Svanovir assay only with additional information about optical density value (data set 2 is a subset of data set 1 ).

${ }^{2}$ Average number and range of daughters per sire.

variable "QNEG" included MAP-questionable animals as a negative outcome. Parameters with both models were estimated using the software package ASREML (Gilmour et al., 2002). For the TM analyses of binary traits, a generalized LM with a probit link function was used. The error variance for the probit model on the underlying scale was equal to 1 (standard normal). For LM and TM analyses, sire models and animal models using the available pedigree information were used. Within the sire models, the results of a model without considering relationship among sires and a model including the relationship between sires from a 2-generation pedigree were compared. Fixed effects included age (in years) and farm, and sire or animal was added as a random effect. Because of the age distribution, 6 age classes were built, with ages $\leq 2,3,4,5,6$, and $>6$ yr. The number of cows within each age class within the 2 data sets is given in Table 1 . Although the MAP status in data set 1 is based on 2 different ELISA tests, this effect was not included in the models. Within each farm, only 1 test was used. Therefore, it was not possible to separate farm and test effects in this data.

Heritabilities using LM or TM sire models were calculated as follows:

$$
h^{2}=\frac{4 \sigma_{s}^{2}}{\sigma_{s}^{2}+\sigma_{e}^{2}}
$$

where $\sigma_{s}^{2}$ and $\sigma_{e}^{2}$ were estimated sire and residual variances, respectively (for the TM probit model, $\sigma_{e}^{2}=1$ ).

To compare the heritability estimates for the binary data assumed to be normally distributed, the estimates on the observed scale were transformed to estimates on the liability scale using the formula of Dempster and Lerner (1950) as follows:

$$
h_{\text {liab }}^{2}=h_{o b s}^{2} \frac{p(1-p)}{z^{2}}
$$

where $h_{o b s}^{2}$ is the heritability on the observed (0/1) scale, $h_{\text {liab }}^{2}$ is the heritability on the liability scale, $p$ is the proportion of MAP-positive animals in the data, and $z$ is the height of the ordinate of the normal distribution corresponding to a truncation point for $p$.

\section{RESULTS}

The frequency of MAP-positive animals varied from 5.0 to $36.9 \%$ in data set 1 and from 6.4 to $22.8 \%$ (data set 2), respectively. For all models, the fixed effects farm and age class significantly affected $\mathrm{ODV}_{\mathrm{T}}$ and MAP status. The estimates of MAP frequency within the age classes are summarized in Figure 1. For QPOS and QNEG, there was an increase in the frequency of positive animals from an age of $\leq 2 \mathrm{yr}$ to an age of $5 \mathrm{yr}$. A slightly lower frequency was estimated for the animals older than 5 yr. For ODV $\mathrm{T}$, a similar trend was observed with animals of 4 and $5 \mathrm{yr}$ showing the largest values and a decrease for animals $\geq 6$ yr old.

The heritability estimates for the 2 data sets and the different traits and models are shown in Table 2. Using sire TM for QPOS or QNEG, both data sets showed greater heritability estimates than from the equivalent animal models. Very similar heritabilities were estimated from sire and animal models from the LM using the observed score (0/1) for QPOS and QNEG. The 
Table 2. Heritability estimates with standard errors for different data sets, traits, and models

\begin{tabular}{|c|c|c|c|c|c|}
\hline \multirow[b]{2}{*}{ Data set } & \multirow{2}{*}{$\begin{array}{l}\text { Method } \\
\text { and trait }\end{array}$} & \multirow[b]{2}{*}{ Model } & \multicolumn{2}{|c|}{ Heritability $^{2}$} & \multirow[b]{2}{*}{$\mathrm{SE}^{2}$} \\
\hline & & & Liability scale & Observed scale & \\
\hline \multirow[t]{5}{*}{1} & TM QPOS & Sire & $0.1274^{\mathrm{b}} / 0.1398^{\mathrm{c}}$ & & $0.0508^{\mathrm{b}} / 0.0534^{\mathrm{c}}$ \\
\hline & TM QNEG & Sire & $0.1145^{\mathrm{b}} / 0.1159^{\mathrm{c}}$ & & $0.0603^{\mathrm{b}} / 0.0626^{\mathrm{c}}$ \\
\hline & TM QNEG & Animal & 0.0519 & & 0.0320 \\
\hline & LM QPOS & Sire & $0.1297^{\mathrm{a}, \mathrm{b}} / 0.1384^{\mathrm{c}}$ & $0.0756^{\mathrm{b}} / 0.0807^{\mathrm{c}}$ & $0.0280^{\mathrm{b}, \mathrm{d}} / 0.0293^{\mathrm{c}, \mathrm{d}}$ \\
\hline & LM QPOS & Animal & $0.1195^{\mathrm{a}}$ & 0.0697 & $0.0241^{\mathrm{d}}$ \\
\hline \multirow[t]{6}{*}{2} & TM QPOS & Sire & $0.1130^{\mathrm{b}} / 0.1267^{\mathrm{c}}$ & & $0.0644^{\mathrm{b}} / 0.0688^{\mathrm{c}}$ \\
\hline & TM QPOS & Animal & 0.0652 & & 0.0347 \\
\hline & TM QNEG & Sire & $0.1380^{\mathrm{b}} / 0.1064^{\mathrm{c}}$ & & $0.0926^{\mathrm{b}} / 0.0861^{\mathrm{c}}$ \\
\hline & TM QNEG & Animal & 0.0518 & & 0.0451 \\
\hline & $\mathrm{LM} \mathrm{ODV} \mathrm{T}_{\mathrm{T}}$ & Sire & $0.0969^{b} / 0.1058^{c}$ & & $0.0483^{b} / 0.0509^{c}$ \\
\hline & $\mathrm{LM} \mathrm{ODV}_{\mathrm{T}}$ & Animal & 0.1018 & & 0.0427 \\
\hline
\end{tabular}

${ }^{1} \mathrm{TM}=$ threshold model; $\mathrm{LM}=$ linear model; $\mathrm{QPOS}=$ animals scored as questionable are added to the Mycobacterium avium ssp. paratuberculosis (MAP)-positive group; QNEG = animals scored as questionable are added to the MAP-negative group; $\mathrm{ODV}_{\mathrm{T}}=$ transformed optical density values.

${ }^{2} \mathrm{a}=$ transformed heritabilities from the observed to the liability scale; $\mathrm{b}=$ sire model without relationship between sires; $\mathrm{c}=$ sire model including relationship between sires; and $d=$ standard error of estimates on the observed scale.

transformed heritabilities to the liability score were slightly greater than the heritability estimates for $\mathrm{ODV}_{\mathrm{T}}$. There was no difference between the sire and animal model in the heritability estimated with the LM for $\mathrm{ODV}_{\mathrm{T}}$. Only very small differences in heritability estimates were found between the sire models with and without relationship information between sires. In most of the sire models including the relationship between sires, slightly greater heritabilities were estimated than in the models without relationship between sires.

All animal TM showed heritabilities between 0.05 and 0.06 for QPOS and QNEG, whereas the estimates for $\mathrm{ODV}_{\mathrm{T}}$ were 0.10 from either sire or animal models, nearly twice as high as in the TM. For QPOS and QNEG, all heritabilities were very similar between data sets within all sire models or all animal models implemented.

\section{DISCUSSION}

The increasing frequency of MAP-positive animals in German Holstein cows with an age up to $5 \mathrm{yr}$ in this study is in agreement with the report of Dreier et al. (2006) in Austrian cattle, mainly including the breeds Austrian Simmental and Brown Swiss. Also, Jubb and Galvin (2000) and Gonda et al. (2006) have shown that cows in later lactations are more likely to produce antibodies to MAP compared with cows in first or second lactation. The smaller number of positive cows $6 \mathrm{yr}$ and older could be biased downwards because of culling of positive cows. Another reason could be the greatest probability of testing positive by ELISA for cows be- tween 2.5 and $4.5 \mathrm{yr}$ of age, as reported by Nielsen and Ersboll (2006).

All estimated heritabilities from the different models in this survey were in the range of estimates from other studies in Holstein cows (Koets et al., 2000; Mortensen et al., 2004; Gonda et al., 2006). The differences in heritability estimates between the sire TM and animal TM, as shown in Table 2, are obvious. RamirezValverde et al. (2001) concluded that there is an advantage of using an animal model rather than a sire model, especially in cases of sires with a small number of daughters.

Following these conclusions, our small average number of daughters ( 7 to 8) for both data sets can probably

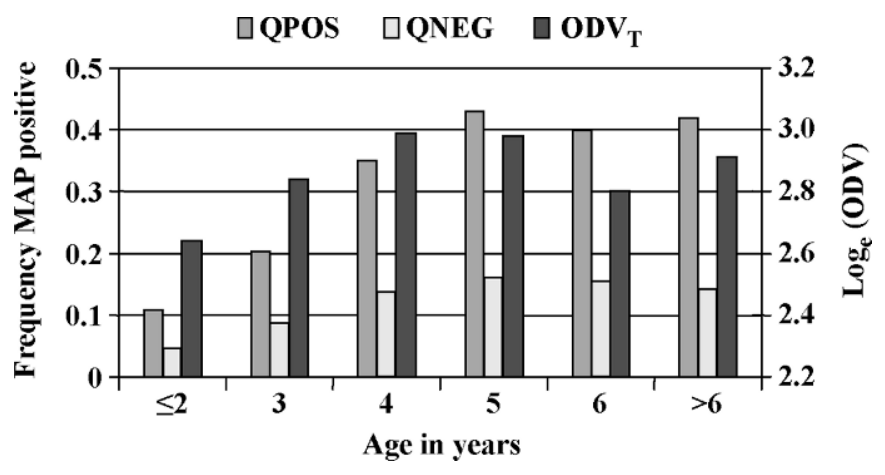

Figure 1. Estimated frequencies of Mycobacterium avium ssp. paratuberculosis (MAP)-positive animals for QPOS (animals scored as questionable added to the MAP-positive group, gray bars) and QNEG (animals scored as questionable added to the MAP-negative group, white bars) and estimates for $\mathrm{ODV}_{\mathrm{T}}$ (optical density value, log-transformed, black bars) for age classes. 
๑ additive genetic variance $\square$ residual variance

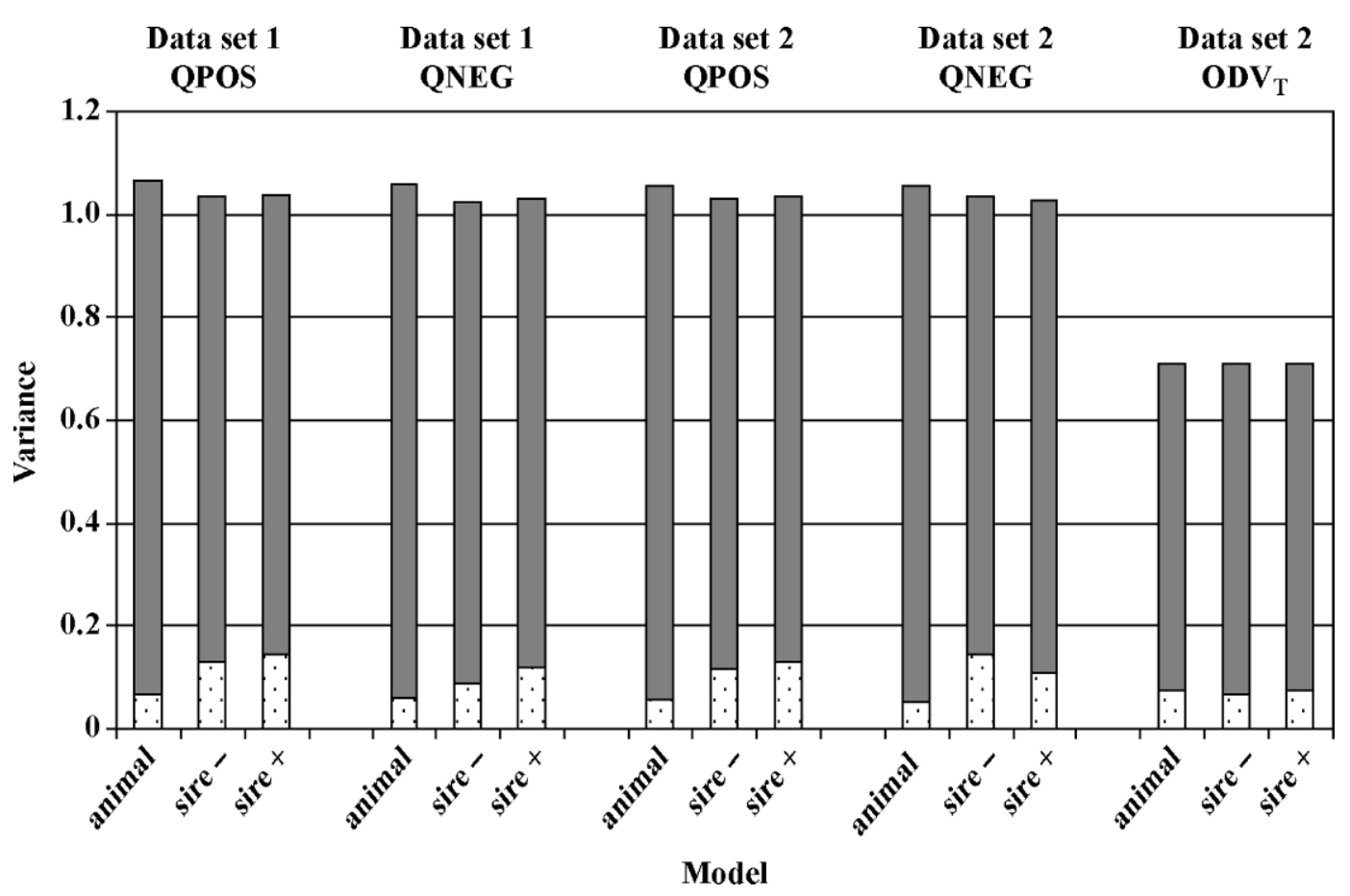

Figure 2. Variance components from the 2 different data sets (data sets 1 and 2) and different models (threshold models for QPOS and QNEG and linear model for $\mathrm{ODV}_{\mathrm{T}}$ ). QPOS = animals scored as questionable added to the Mycobacterium avium ssp. paratuberculosis (MAP)positive group; QNEG = animals scored as questionable added to the MAP negative group; and $\mathrm{ODV}_{\mathrm{T}}$ (optical density value, log-transformed).

explain the differences in heritability estimates between sire and animal TM. The slightly larger estimates from the animal LM than from the animal TM are also in agreement with Ramirez-Valverde et al. (2001) and Hagger and Hofer (1989), both indicating that LM are robust toward departures from normality, especially in cases with more equal probabilities for both cases ( 0 and 1$)$. The robustness of LM vs. TM is probably also an explanation for the small differences between sire and animal LM in the present study. The small differences in heritability estimates between the 2 data sets analyzed here show that the smaller data set of only 2,084 animals can be assumed to be a representative sample of all available data. This is also shown in Table 1, with similar proportions of MAP positive, MAP negative, and questionable animals. No convergence problems occurred with either sire or animal models, as reported by Koets et al. (2000) or Janss and Bolder (2000).

The applied ELISA tests in the study use different antigens. The IDEXX test detects antibodies to protoplasmic MAP antigens and includes a preabsorption step with Mycobacterium phlei, whereas the Svanovir test is a nonabsorbed ELISA and detects antibodies to lipoarabinomannan. Test prevalence was greater in herds tested with Svanovir ELISA, presumably because of lower specificity. As explained in the data description, the difference between the test methods, as shown in Table 1, is probably also biased through farm effects because of the confounding between farm and test used. Therefore, we could not compare prevalences for both tests within each herd or for each animal. The results from the 2 data sets also show that the expected differences between the 2 ELISA tests in the detection of the number of positive animals did not influence the heritability estimates. This is probably a sign of good genetic connectedness between farms.

Hoeschele and Tier (1995) and Moreno et al. (1997) found biased estimates from animal models compared with sire models for threshold traits, especially in data sets with a large number of fixed effect classes. In the present data set, only fixed effects with a low number of classes and without small numbers of observations with either score 0 or score 1 in some classes were analyzed, which is a possible explanation for the stable and similar results from sire and animal models in this study.

To further explain the differences between sire and animal models, TM or LM, the estimated variance components from different models and data sets are shown 
additive genetic variance

Data set 1

QPOS linear model residual variance

Data set 1 QNEG linear model

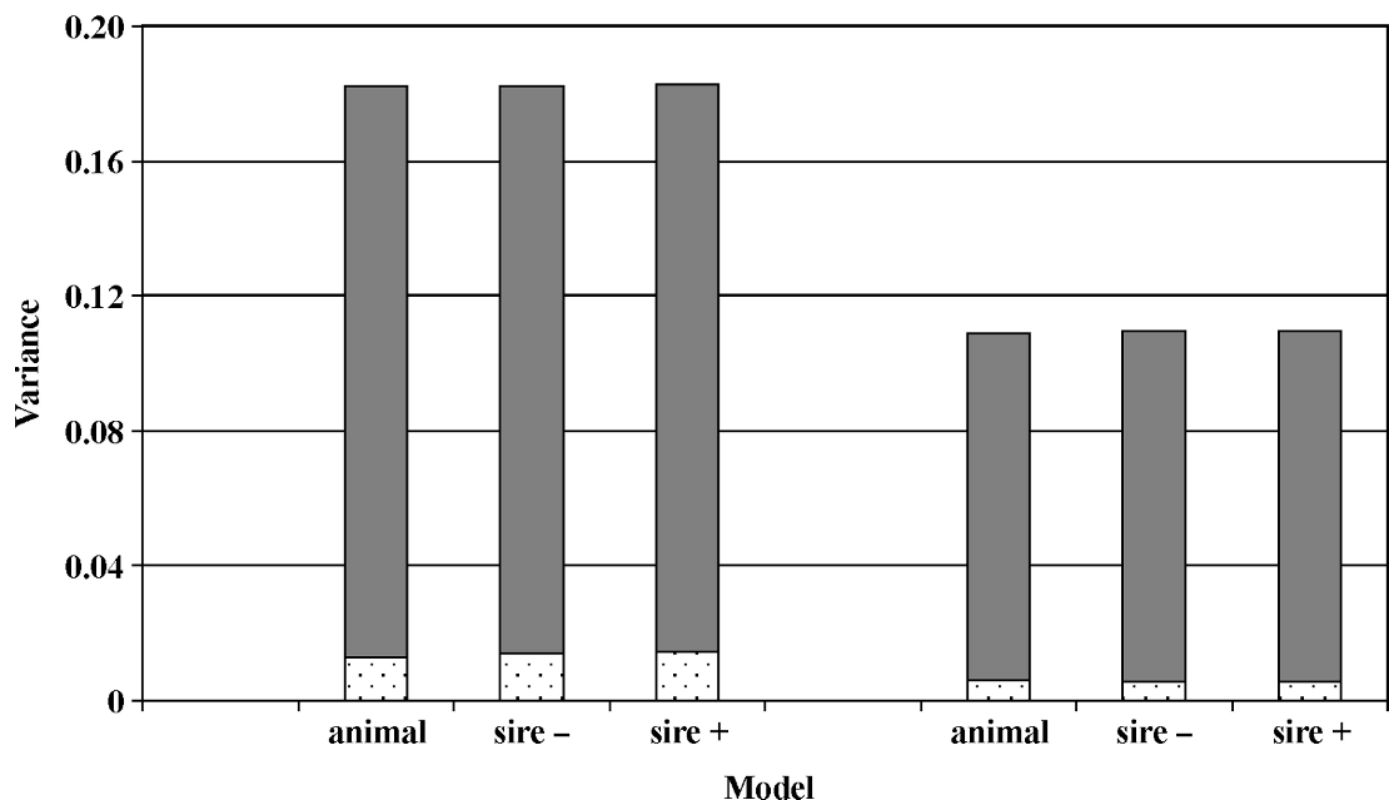

Figure 3. Additive and residual variances estimated with linear sire and animal models from data set 1. QPOS = animals scored as questionable added to the Mycobacterium avium ssp. paratuberculosis (MAP)-positive group; QNEG = animals scored as questionable added to the MAP negative group.

in Figures 2 and 3. In both figures, the residual variance shown for the sire models was calculated as the total variance (sire variance plus 1 ) minus the additive genetic variance (4 times sire variance). Applying TM, the variance components cannot be directly compared between animal models, because the error variance using the probit function is set to be 1 . In the sire model, this includes the residual error plus three-fourths of the additive genetic variance, whereas it includes only the error variance in the animal model. This factor had an effect on the total variance estimated from both models, with the lower total variance obtained using sire models, as can be seen in Figure 2. This effect was not expected when implementing the linear models for $\mathrm{ODV}_{\mathrm{T}}$ or for QPOS and QNEG, as is shown in Figure 2 and Figure 3, respectively. Although the total variance in sire TM was expected to be smaller than in animal models, the proportion of additive genetic to total variance should not be different between models. One would expect to obtain a smaller estimate of additive genetic variance in the sire models than in the animal model. Figure 2 clearly shows that the additive genetic variance is greater in all sire TM than in the animal model, which resulted in the overestimated heritabilities.
The conclusions from Ramirez-Valverde et al. (2001) that sire models in cases of small number of progeny for sires will show larger errors for the estimates and the fact that this error is multiplied by 4 for the calculation of the additive genetic variance are possible explanations for the overestimation of heritabilities using sire models for these data. A sire model assumes that all progeny of a sire are half-sibs with an average relationship of one-fourth, which can only occur when all dams mated to a sire are unrelated. This assumption is probably not valid in the analyzed data sets, which also resulted in an overestimation of the genetic variance. The additional consideration of relationship between sires in the sire models did not yield results different from the sire models without accounting for relationships between sires. The general tendency is that with the additional pedigree information, a slightly greater additive genetic variance is estimated, which could probably be explained by better separation between genetic and environmental effects, through better connectedness over the farms, for example. These models could definitely not solve the problem of overestimation caused by the average relationship between daughters within sires being greater than one-fourth. 
When one has available both the OD measurements of the ELISA test and the MAP status (positive or negative), the transformed OD measurement should be used for parameter estimation and genetic evaluation for antibody response to MAP. For $\mathrm{ODV}_{\mathrm{T}}$, sire and animal models yielded very similar heritability estimates, in contrast to sire or animal TM.

Similar to the study of Mortensen et al. (2004), the estimated heritability in this study does not provide information about genetic variation in the clinical disease or about the possibility of shedding of mycobacteria. Furthermore, one cannot discern whether negative-tested animals are not detected but actually infected or truly not infected because they did not have contact with MAP in the susceptible period of life. The number of such false-negative animals is probably highly dependent on the prevalence of MAP within farms. This problem would be partly overcome through the development of more sensitive tests for MAP detection. The changes in immune response from a proinflammatory response to antibody production are often associated with progression to clinical disease (Coussens, 2004). Therefore, selection for animals that do not develop antibodies would be indicated in MAP control programs. On the other hand, these animals could be infected and shed MAP in their feces, even though they do not manifest clinical disease. So, selection of these animals is inappropriate for promoting selection programs. Additional, more informative genetic parameters need to be identified for development of efficient MAP breeding strategies. In addition, different MAP strains may trigger different pathways and subsequent differences in host immune responses to MAP. This could influence herd prevalences and should be considered in future analyses.

\section{CONCLUSIONS}

The study confirms a low genetic background for antibody response to MAP in dairy cows, with heritability estimates between 0.05 and 0.13 from the different models and data sets. With the data structure in this study, with a high number of sires with an average of 8 daughters, sire TM overestimated genetic variance compared with animal TM. Using the OD values of the Svanovir ELISA test as normal distributed trait (logtransformed) led to very similar heritability estimates of about 0.1 from sire and animals models. The heritability of around 0.1 indicates that data from ELISAbased diagnostic tests for Johne's disease could be used as a selection criterion in breeding programs, although the expected genetic progress will be slow. Further studies on possible candidate genes for paratuberculo- sis in cattle could help in selection against Johne's disease and improve possible selection response.

\section{ACKNOWLEDGMENTS}

The authors would like to thank A. Bünger (Vereinigte Informationssysteme Tierhaltung w. V., Verden, Germany) for providing the pedigree information and S. Horner from the Animal Health Service of Thuringia for providing the ELISA test data.

\section{REFERENCES}

Chiodini, R. J., H. J. Van Kruiningen, and R. S. Merkal. 1984. Ruminant paratuberculosis (Johne's disease): The current status and future prospects. Cornell Vet. 74:218-262.

Clarke, C. J. 1997. The pathology and pathogenesis of paratuberculosis in ruminants and other species. J. Comp. Pathol. 116:217-261.

Coussens, P. M. 2004. Model for immune responses to Mycobacterium avium subspecies paratuberculosis in cattle. Infect. Immun. 72:3089-3096.

Dekkers, J. C., and F. Hospital. 2002. The use of molecular genetics in the improvement of agricultural populations. Nat. Rev. Genet. $3: 22-32$.

Dempster, E. R., and I. M. Lerner. 1950. Heritability of threshold characters. Genetics 35:212-235.

Dreier, S., J. L. Khol, B. Stein, K. Fuchs, S. Gutler, and W. Baumgartner. 2006. Serological, bacteriological and molecular biological survey of paratuberculosis (Johne's disease) in Austrian cattle. J. Vet. Med. B Infect. Dis. Vet. Public Health 53:477-481.

Gasteiner, J., H. Wenzl, K. Fuchs, U. Jark, and W. Baumgartner. 1999. Serological cross-sectional study of paratuberculosis in cattle in Austria. J. Vet. Med. B Infect. Dis. Vet. Public Health 46:457-466.

Gilmour, A. R., B. J. Gogel, B. R. Cullis, S. J. Welham, and R. Thompson. 2002. ASReml User Guide Release 1.0 VSN International Ltd., Hemel Hempstead, UK.

Gonda, M. G., Y. M. Chang, G. E. Shook, M. T. Collins, and B. W. Kirkpatrick. 2006. Genetic variation of Mycobacterium avium ssp. paratuberculosis infection in US Holsteins. J. Dairy Sci. 89:1804-1812.

Gonda, M. G., B. W. Kirkpatrick, G. E. Shook, and M. T. Collins. 2007. Identification of a QTL on BTA20 affecting susceptibility to Mycobacterium avium ssp. paratuberculosis infection in US Holsteins. Anim. Genet. 38:389-396.

Goodger, W. J., M. T. Collins, K. V. Nordlund, C. Eisele, J. Pelletier, C. B. Thomas, and D. C. Sockett. 1996. Epidemiologic study of on-farm management practices associated with prevalence of $M y$ cobacterium paratuberculosis infections in dairy cattle. J. Am. Vet. Med. Assoc. 208:1877-1881.

Hacker, U., K. Huttner, and M. Konow. 2004. Investigation of serological prevalence and risk factors of paratuberculosis in dairy farms in the state of Mecklenburg-Westpommerania, Germany. Berl. Münch. Tierärztl. Wschr. 117:140-144.

Hagger, C., and A. Hofer. 1989. Correlations between breeding values of dairy sires for frequency of dystocia evaluated by a linear and non linear method. J. Anim. Sci. 67(Suppl. 1):88. (Abstr.)

Heringstad, B., Y. M. Chang, D. Gianola, and G. Klemetsdal. 2005. Genetic analysis of clinical mastitis, milk fever, ketosis, and retained placenta in three lactations of Norwegian red cows. J. Dairy Sci. 88:3273-3281.

Hinger, M., H. Brandt, S. Horner, and G. Erhardt. 2007. Short communication: Association analysis of microsatellites and Mycobacterium avium subspecies paratuberculosis antibody response in German Holsteins. J. Dairy Sci. 90:1957-1961.

Hoeschele, I., and B. Tier. 1995. Estimation of variance components of threshold characters by marginal posterior modes and means via Gibbs sampling. Genet. Sel. Evol. 27:519-540. 
Jakobsen, M. B., L. Alban, and S. S. Nielsen. 2000. A cross-sectional study of paratuberculosis in 1155 Danish dairy cows. Prev. Vet. Med. 46:15-27.

Janss, L. L. G., and N. M. Bolder. 2000. Heritabilities of and genetic relationships between salmonella resistance traits in broilers. J. Anim. Sci. 78:2287-2291.

Jubb, T., and J. Galvin. 2000. Herd testing to control bovine Johne's disease. Vet. Microbiol. 77:423-428.

Koets, A. P., G. Adugna, L. L. Janss, H. J. van Weering, C. H. Kalis, G. H. Wentink, V. P. Rutten, and Y. H. Schukken. 2000. Genetic variation of susceptibility to Mycobacterium avium subsp. paratuberculosis infection in dairy cattle. J. Dairy Sci. 83:2702-2708.

Moreno, C., D. Sorensen, L. A. Garcia Cortes, L. Varona, and J. Altarriba. 1997. On biased inferences about variance components in the binary threshold model. Genet. Sel. Evol. 29:145-160.

Mortensen, H., S. S. Nielsen, and P. Berg. 2004. Genetic variation and heritability of the antibody response to Mycobacterium avium subspecies paratuberculosis in Danish Holstein cows. J. Dairy Sci. 87:2108-2113.

Muskens, J., H. W. Barkema, E. Russchen, K. van Maanen, Y. H. Schukken, and D. Bakker. 2000. Prevalence and regional distribution of paratuberculosis in dairy herds in the Netherlands. Vet. Microbiol. 77:253-261.

Muskens, J., A. R. Elbers, H. J. van Weering, and J. P. Noordhuizen. 2003. Herd management practices associated with paratuberculo- sis seroprevalence in Dutch dairy herds. J. Vet. Med. B Infect. Dis. Vet. Public Health 50:372-377.

NAHMS. 1997. Johne's disease on U.S. dairy operations. USDA:APHIS:VS, CEAH, National Animal Health Monitoring System, Fort Collins, CO.

Nielsen, S. S., and A. K. Ersboll. 2006. Age at occurrence of Mycobacterium avium subspecies paratuberculosis in naturally infected dairy cows. J. Dairy Sci. 89:4557-4566.

Ott, S. L., S. J. Wells, and B. A. Wagner. 1999. Herd-level economic losses associated with Johne's disease on US dairy operations. Prev. Vet. Med. 40:179-192.

Raadsma, H., and K. Fullard. 2006. QTL mapping and Gene Markers for Resistance to Infectious Diseases in Sheep and Cattle. 8th World Congr. Genet. Appl. Livest. Prod., Belo Horizonte, MG, Brazil. Commun. No.15-01.

Ramirez-Valverde, R., I. Misztal, and J. K. Bertrand. 2001. Comparison of threshold vs. linear and animal vs. sire models for predicting direct and maternal genetic effects on calving difficulty in beef cattle. J. Anim. Sci. 79:333-338.

Snowder, G. D., L. D. Van Vleck, L. V. Cundiff, and G. L. Bennett. 2005. Influence of breed, heterozygosity, and disease incidence on estimates of variance components of respiratory disease in preweaned beef calves. J. Anim. Sci. 83:1247-1261.

Sweeney, R. W. 1996. Transmission of paratuberculosis. Vet. Clin. North Am. Food Anim. Pract. 12:305-312. 